\title{
Efecto del cambio en el camino de deformación en la formabilidad de chapas de acero DP-780 y zinc Zn20
}

\author{
Effect of strain-path change on the formability \\ of DP-780 steel and Zn20 zinc sheets
}

\author{
Claudio Daniel Schwindt ${ }^{1}$, Fernando Schlosser ${ }^{1,2}$, \\ Javier Walter Signorelli ${ }^{2}$
}

\footnotetext{
${ }^{1}$ Departamento de Ingeniería, UNS - CONICET, Av. Alem 1253, Bahía Blanca, Buenos Aires, Argentina. e-mail: claudio.schwindt@uns.edu.ar

${ }^{2}$ División Materiales, IFIR-UNR-CONICET, Bv. 27 de Febrero 210b, Rosario, Santa Fe, Argentina. e-mail: schlosser@ifir-conicet.gov.ar; signorelli@ifir-conicet.gov.ar
}

\section{RESUMEN}

Los Diagramas Límite de Conformado (DLC), frecuentemente utilizados para caracterizar la formabilidad de chapas metálicas, se determinan asumiendo trayectorias de carga proporcionales hasta la ocurrencia de la inestabilidad plástica. Sin embargo, las deformaciones límite son afectadas significativamente por los cambios en los caminos de deformación, práctica muy usual en procesos reales de conformado de metales. El objetivo de este trabajo es analizar el efecto de la trayectoria de carga sobre el DLC de chapas de acero DP780 de $0,9 \mathrm{~mm}$ de espesor y zinc Zn20 de 0,65 mm de espesor utilizando ensayos en dos etapas. Se estudiaron las deformaciones límite bajo tracción uniaxial para los materiales pre-deformados bajo carga equibiaxial. El DLC de referencia se obtuvo a través de ensayos de acopado hidráulico y de tracción uniaxial en probetas con diferentes geometrías de entalla. Las deformaciones límite obtenidas en la chapa DP-780 pre-deformada se encuentran por encima del diagrama de referencia, evidenciando una no despreciable dependencia de la formabilidad del material con la historia de carga. Por otro lado, las diferencias entre los DLCs de la chapa Zn20 son significativas. Esto se atribuye no sólo a efectos de trayectoria sino también a la recristalización dinámica continua que experimenta el material a temperatura ambiente.

Palabras clave: Formabilidad, chapas metálicas, cambios de trayectoria, DP-780, Zn20.

\begin{abstract}
The Forming Limit Diagram (FLD), frequently used to characterize the formability of sheet metals, is determined by assuming proportional loading histories up to the onset of necking. However, the limit strains are highly affected by strain-paths changes, which are very usual in real metal forming processes. The aim of this work is to analyze the effect of the loading history on the FLDs of a 0,9 mm thick DP-780 steel sheet and a $0,65 \mathrm{~mm}$ thick Zn20 zinc sheet by applying two-stage tests. The forming limits are studied under uniaxial deformation for the biaxially pre-deformed materials. The reference FLD was obtained by hydraulic bulge tests and uniaxial tests with different samples geometries. The limit strains of the pre-deformed DP-780 steel sheet are above the reference FLD, which reveals a non-negligible dependency between the material formability and the loading history. On the other hand, the differences between the FLDs of the zinc sheet are significant. This is attributed not only to the effect of changes in the strain path, but also to the continuous dynamic recrystallization experienced by the material at room temperature.
\end{abstract}

Keywords: Formability, sheet metals, strain-path change, DP-780, Zn20.

\section{INTRODUCCIÓN}

El Diagrama Límite de Conformado (DLC) es una herramienta utilizada para determinar si las deformaciones sobre un producto estampado se encuentran en zona segura o presentan riesgo de falla. La separación entre estas zonas está delimitada por una línea comúnmente denominada Curva Límite de Conformado (CLC), determinada mediante una amplia variedad de ensayos experimentales. Debido a su simpleza, el DLC se ha 
vuelto esencial para evaluar la conformabilidad de chapas metálicas así como para el diseño y/o comprobación de procesos industriales. Sin embargo, el comportamiento plástico de los materiales depende fuertemente de la historia de carga, en particular, del camino de deformación específico. En muchas operaciones de conformado, las chapas son deformadas bajo diferentes matrices y etapas, experimentando variaciones en los caminos de deformación. Por lo tanto, es importante investigar la influencia de estos cambios de trayectorias sobre el comportamiento límite del material.

Muchos autores han demostrado que las cargas no proporcionales poseen una importante influencia en el nivel y la forma de las CLCs [1], lo que puede llevar a interpretar erróneamente la severidad de conformado en procesos de múltiples etapas. VOLK et al. [2] introdujeron un nuevo método para describir el comportamiento de falla de materiales en operaciones de conformado realizadas en dos etapas y validaron sus resultados en chapas de acero dual-phase HC300X y HC450X. SUTTNER y MERKLEIN [3] analizaron el efecto de una pre-deformación biaxial sobre las propiedades mecánicas y la formabilidad de una aleación de aluminio AA5182 y una chapa de acero DP600 sometidas a solicitaciones de corte puro. Ambos materiales mostraron un aumento en las deformaciones límite alcanzadas a medida que se aumentaba el nivel de predeformación. LEOTOING y GUINES [4] desarrollaron un dispositivo tipo crucifijo biaxial para estudiar la influencia de cambios de camino de deformación sobra la formabilidad de chapas en un único ensayo, sin necesitar la descarga del material. Resultados sobre una chapa de aluminio AA5086 mostraron que los valores de deformación límite son fuertemente influenciados por el nivel de pre-deformación, que puede tanto mejorar como empeorar la formabilidad de este material. GRAF y HOSFORD [5,6] estudiaron la influencia del cambio en los caminos de deformación en chapas de aluminio AA2008 y AA6111-T4 usando predeformaciones uniaxiales, de deformación plana y equibiaxiales. Los resultados mostraron que, en los mencionados materiales, el pre-estirado biaxial generalmente disminuye la CLC, mientras que una predeformación uniaxial incrementa la CLC en el sector derecho del diagrama sin encontrar un efecto significativo sobre el sector izquierdo del mismo. Estos resultados fueron numéricamente verificados utilizando el modelo de Marciniak-Kuczynski (MK) en conjunto con criterios de fluencia anisótropos [7,8]. SCHWINDT et al. [9] estudiaron la influencia de cambios abruptos de trayectorias de carga para materiales FCC isotrópicos y con textura de laminado, combinando la técnica MK conjuntamente con un modelo de plasticidad cristalina (VPSC - Visco-Plastic Self-Consistent). Los resultados mostraron diferentes comportamientos: aumento de formabilidad para pre-deformaciones uniaxiales seguidas de estirado equibiaxial; disminución de las deformaciones límite para estirado biaxial seguido de deformación plana y un comportamiento intermedio para trayectorias biaxiales seguidas de tracción uniaxial.

El objetivo de este trabajo es determinar el efecto de la trayectoria de carga sobre el DLC de chapas de acero DP-780 y zinc Zn20, utilizando ensayos en dos etapas. Se estudian las deformaciones límite bajo tracción uniaxial para los materiales pre-deformados bajo carga equibiaxial mediante ensayos Marciniak. El DLC de referencia se obtiene a través de ensayos de acopado hidráulico y de tracción uniaxial en probetas con diferentes geometrías de entalla. Se discutirán los comportamientos dispares entre ambos materiales.

\section{MATERIALES Y MÉTODOS}

En la presente investigación se estudia un acero DP-780 y una chapa de zinc Zn20 de 0,9 mm y 0,65 mm de espesor, respectivamente. Seguidamente se presenta la caracterización microestructural y mecánica de cada material, así como las metodologías empleadas para determinar el DLC para trayectorias tanto lineales como bilineales.

\subsection{Caracterización microestructural y mecánica}

Para poder visualizar las características microestructurales de los materiales se utilizó un microscopio óptico Leica DM ILM. El ataque químico para la chapa de zinc se realizó mediante una solución de ácido crómico $\left(\mathrm{CrO}_{3}\right)$, sulfato de sodio $\left(\mathrm{Na}_{2} \mathrm{SO}_{4}\right)$ y agua, durante 10 segundos. En la micrografía (Figura 1, Izquierda) se observa la presencia del compuesto intermetálico $\mathrm{TiZn}_{16}$, el cual presenta contraste negro y forma alargada en la dirección de laminado (RD). Por otro lado, el ataque químico del acero DP-780 se realizó en dos etapas mediante los reactivos Vilella y Nital 2\%, en un tiempo aproximado de 10 segundos en cada etapa. La micrografía (Figura 1, Derecha) muestra a los granos de martensita, de color más claro, conectados entre ellos formando una estructura de red de tipo collar, distribuidos uniformemente sobre los bordes de grano de la ferrita. 

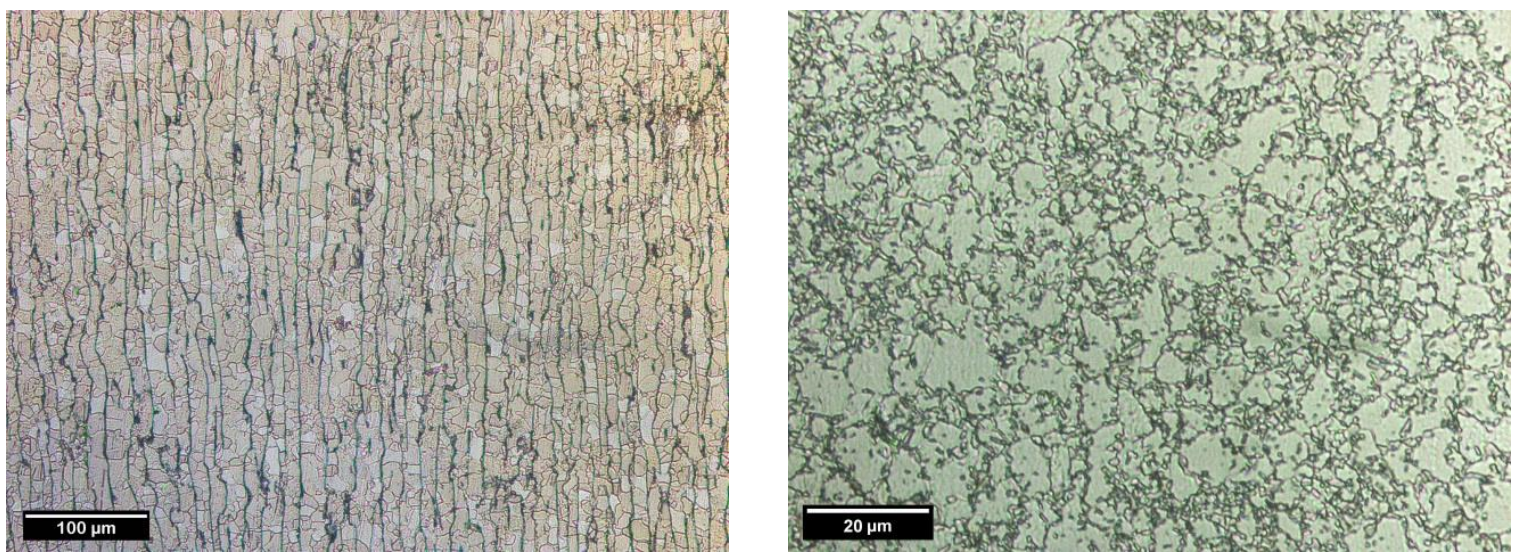

Figura 1: Micrografías ópticas del plano de las chapas con la dirección de laminación alineada verticalmente: (Izquierda) Zn20 (200x); (Derecha) DP-780 (1000x).

Las propiedades mecánicas de los materiales fueron medidas siguiendo la norma ASTM E8-04 usando probetas planas de dimensiones reducidas (subsize), cortadas a lo largo de la dirección de laminado. Los parámetros de endurecimiento correspondientes a la ley de Hollomon $\left(\sigma=K \varepsilon^{n}\right)$ se obtienen a partir de aproximar las curvas de tensión-deformación verdadera sobre los siguientes rangos de deformación: $[0,05-0,12]$ para DP-780, y [0,05-0,15] para Zn20. Los ensayos fueron desarrollados a temperatura ambiente utilizando una máquina de ensayo universal Instron 5989 de $150 \mathrm{kN}$ de capacidad. Las probetas de acero DP-780 fueron cargadas con control de desplazamiento a una velocidad del cabezal de $1,5 \mathrm{~mm} / \mathrm{min}$, dando una velocidad de deformación de aproximadamente $10^{-3} \mathrm{~s}^{-1}$. Para la aleación de zinc los ensayos de tracción fueron llevados a cabo a una velocidad de deformación constante de $5 \times 10^{-4} \mathrm{~s}^{-1}$. Se obtuvieron datos de al menos dos ensayos válidos y los resultados fueron promediados. Las curvas de carga ingenieriles obtenidas se muestran en la Figura 2, mientras que las propiedades mecánicas medidas se resumen en la Tabla 1.

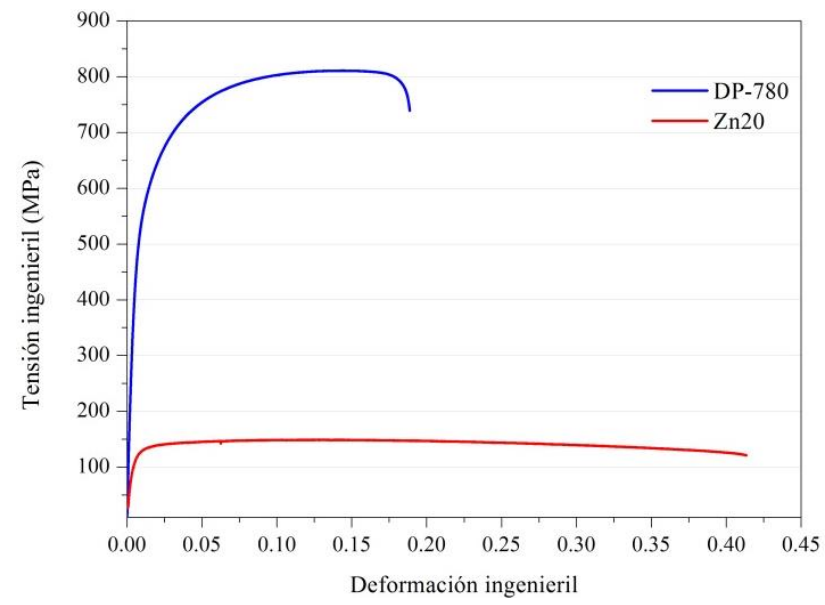

Figura 2: Curvas de carga ingenieriles correspondientes al acero DP-780 y al zinc Zn20 medidas sobre RD.

Tabla 1: Propiedades mecánicas de los materiales medidas sobre la dirección de laminado.

\begin{tabular}{l|l|l|l|l}
\hline MATERIAL & $\boldsymbol{\sigma}_{0,2} \%(\mathrm{MPa})$ & $\mathbf{U T S}(\mathrm{MPa})$ & $\boldsymbol{K}(\mathbf{M P a})$ & $\boldsymbol{n}$ \\
\hline DP-780 & 494 & 812,6 & 1279 & 0,157 \\
\hline Zn20 & 98,5 & 148,9 & 128,6 & 0,113 \\
\hline
\end{tabular}

\subsection{Diagrama Límite de Conformado}

Actualmente existen variadas técnicas experimentales para determinar el DLC, las cuales incluyen diferentes geometrías de herramientas y de probetas para deformar el material bajo diferentes trayectorias de deformación, nominalmente proporcionales, en el espacio de deformaciones principales. Dichos caminos recorren 
estados desde tracción uniaxial hasta tracción biaxial equilibrada, pasando por deformación plana. En el presente trabajo la determinación del sector izquierdo del DLC se realizó mediante ensayos de tracción sobre probetas con diferentes geometrías de entallas, mientras que las deformaciones límites en el sector derecho del mismo se obtuvieron a través de ensayos de acopado hidráulico.

Los ensayos de tracción constaron de la utilización de dos geometrías de probetas para lograr condiciones de deformación uniaxial simple y deformación plana (Figura 3, Izquierda), todas orientadas en la dirección de laminación. Para el acero DP-780, la velocidad de desplazamiento del cabezal de la máquina fue de 0,5 y $0,2 \mathrm{~mm} / \mathrm{min}$ para las geometrías de tracción uniaxial y deformación plana, mientras que para el zinc $\mathrm{Zn} 20$ fue de 3,8 y $0,5 \mathrm{~mm} / \mathrm{s}$, respectivamente.
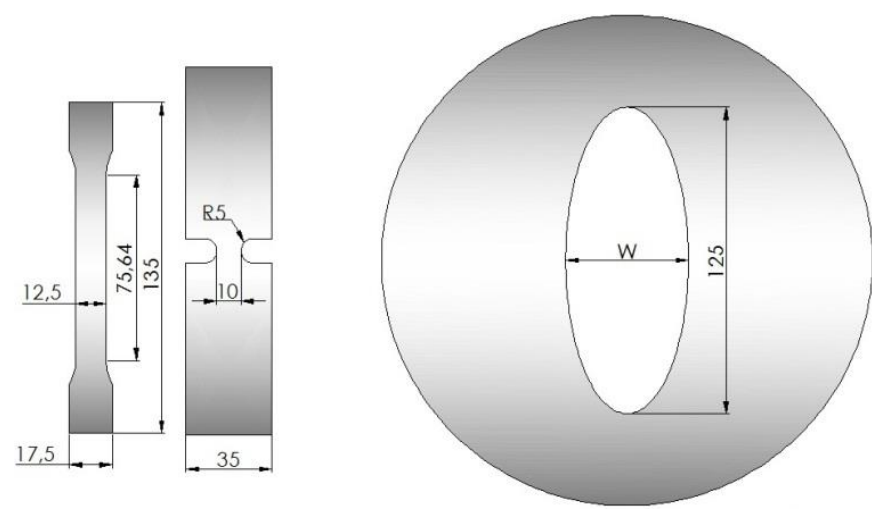

Figura 3: Geometría de las muestras utilizadas en la determinación del DLC: (Izquierda) ensayos de tracción uniaxial y deformación plana, respectivamente; (Derecha) ensayos de acopado hidráulico, donde W =42mm; $83 \mathrm{~mm}$; $97 \mathrm{~mm}$ y 125 $\mathrm{mm}$.

El ensayo de acopado hidráulico es ampliamente conocido en formabilidad para producir un estado de estirado biaxial, y es mayormente utilizado para ensayar chapas delgadas ya que las tensiones debidas a flexión pueden ser despreciadas. El procedimiento experimental consiste en deformar las muestras ancladas entre la matriz y el sujetador utilizando un líquido presurizado. Incrementando continuamente la presión, la probeta es estirada biaxialmente y deformada hasta la ruptura. Para la obtención de diferentes trayectorias de deformación se emplearon diferentes relaciones de aspecto de elipse de la matriz (Figura 3, Derecha). Las probetas son de sección circular con un diámetro de $200 \mathrm{~mm}$. La velocidad de deformación del material queda determinada por el caudal de flujo de la bomba de desplazamiento positivo hacia la matriz, resultando en valores del orden de $1 \times 10^{-3} \mathrm{~s}^{-1}$ y $0,05 \mathrm{~s}^{-1}$ para las chapas de DP-780 y Zn20, respectivamente. En todos los casos, los ensayos fueron realizados a temperatura ambiente.

Las deformaciones fueron medidas mediante correlación digital de imágenes. Ésta es una técnica óptica, de campo completo, que consiste en la toma de imágenes digitales durante el ensayo de una probeta desde su estado inicial (considerado como el de referencia) hasta su estado final (deformado) de modo de medir desplazamientos y deformaciones sobre la superficie analizada [10]. Previamente a la realización del ensayo, se aplica una base de pintura blanca, flexible y adherente, sobre la superficie de la muestra, y posteriormente se genera un moteado aleatorio de color negro, también llamado patrón de speckle, para generar el máximo contraste posible. Comparando las imágenes adquiridas, el software de correlación de imágenes divide la zona de análisis sobre la probeta en subconjuntos virtuales, denominados facetas y, mediante la aplicación de algoritmos de correlación, busca una región o faceta de la imagen de referencia en la imagen deformada, determinando el vector de desplazamientos en cada conjunto de facetas procesadas y a partir de ellos es capaz de obtener los campos de deformaciones. Para los ensayos de tracción es suficiente la utilización de la técnica en dos dimensiones (2D), con lo cual sólo se requiere una única fotografía, y por ende una única cámara fotográfica, por cada estado de deformación deseado. Por otra parte, cuando se requiere medir deformaciones sobre superficies curvadas (3D) de ensayos fuera del plano, como el ensayo de acopado hidráulico, se utiliza un arreglo de dos cámaras. Se toman en este caso dos fotografías (una por cada cámara) de cada estado (original y deformado). Las dos cámaras permiten localizar cualquier punto en el espacio tridimensional dado por la superficie de la probeta a partir de su posición en ambas imágenes.

Para determinar los valores de deformación límite de cada ensayo se siguió una metodología similar a la propuesta por BRAGARD et al. [11]. Dicho procedimiento consiste en evaluar la distribución de deformaciones principales a lo largo de varias secciones perpendiculares a la grieta o estricción localizada en la 
muestra. En cada sección se realiza el ajuste de la distribución de deformación principal máxima empleando una parábola de orden 4. Originalmente, Bragard utilizó únicamente un polinomio de grado 2, sin embargo, resultados obtenidos por SERENELLI [12] muestran que los polinomios de grado 4 permiten conseguir una mejor descripción del fenómeno. Los puntos sobre la estricción o fractura son convenientemente descartados del ajuste y la deformación límite mayor en cada sección se corresponde con el valor máximo de la función ajustada sobre los puntos restantes. Para definir la deformación límite menor se debe ajustar otro polinomio a las deformaciones menores e intersectarlo con la abscisa correspondiente al máximo de la curva polinomial anterior. Finalmente, la media de los valores obtenidos en cada sección analizada corresponde al punto sobre la CLC.

\subsection{Trayectorias bilineales}

Para estudiar la influencia de los cambios en las trayectorias de deformación sobre la formabilidad de ambos materiales se llevaron a cabo ensayos en dos etapas de deformación, tal como se esquematiza en la Figura 4 (Izquierda). El pre-estirado inicial se realiza mediante ensayos Marciniak con muestras circulares de $200 \mathrm{~mm}$ de diámetro, lo que impone condiciones de deformación equibiaxial a la muestra. En este ensayo la carga es transferida a la probeta mediante un punzón plano a través de una máscara de chapa, la cual posee un orificio central en la zona de interés de $32 \mathrm{~mm}$, eliminando los efectos de fricción en esta región de la muestra. Los ensayos se llevaron a cabo en una prensa hidráulica Adabor de 120 Tm. La matriz Marciniak posee un diámetro de $119 \mathrm{~mm}$, un radio de empalme de 6,35 $\mathrm{mm}$ y dispone de un freno de $4 \mathrm{~mm}$ de altura, ubicado sobre un diámetro de $153 \mathrm{~mm}$. El punzón es de $100 \mathrm{~mm}$ de diámetro y dispone del mismo radio de empalme que la matriz. Las condiciones de fricción en las interfaces punzón-máscara y máscara-probeta son claves para el éxito del ensayo. En el primero de los casos la fricción debe ser mínima, siendo lo contrario en el segundo. Con tal objetivo, se interpone una fina capa de $\mathrm{MoS}_{2}$ y un disco delgado de Politetrafluoroetileno (PTFE) de 0,7 mm de espesor entre el punzón y la máscara, mientras que no se utiliza lubricación entre la máscara y la chapa.
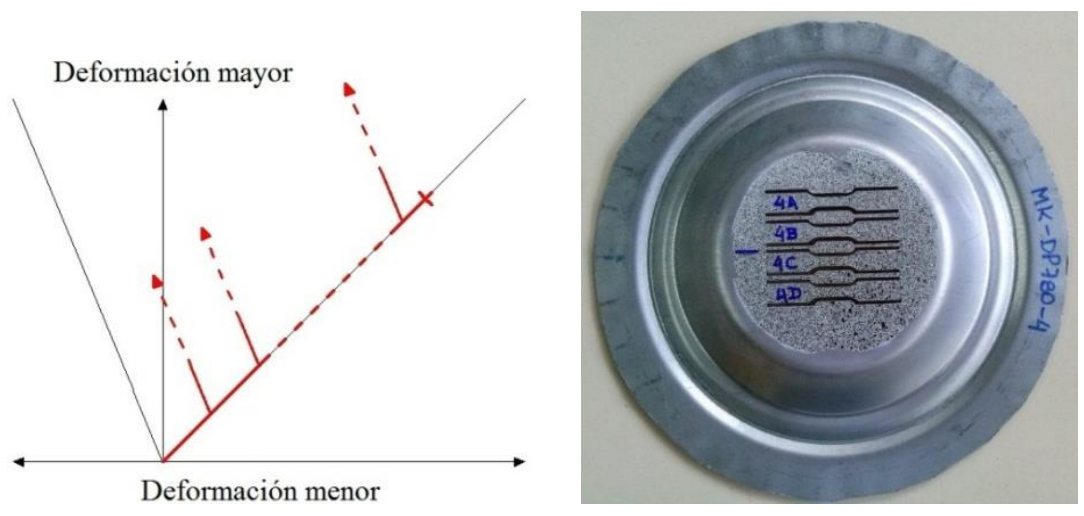

Figura 4: (Izquierda) Trayectorias bilineales investigadas; (Derecha) extracción de muestras de tracción uniaxial a partir de una probeta pre-deformada mediante un ensayo Marciniak.

Luego del ensayo Marciniak se extraen muestras pequeñas de tracción uniaxial de la superficie predeformada alineadas con la dirección de laminación de la chapa tal como muestra la Figura 4 (Derecha). El corte de las mismas se realiza por medio de electroerosión. Seguidamente se realizan ensayos de tracción uniaxial hasta alcanzar la presencia de estricción localizada o fractura de los materiales. Se siguió la misma metodología del apartado anterior para determinar los valores de deformación límite en las muestras.

\section{RESULTADOS Y DISCUSIÓN}

Los DLCs experimentales, obtenidos a partir de trayectorias de deformación lineales, se muestran en la Figura 5. Las deformaciones principales menores se grafican a lo largo del eje $x$ y las mayores sobre el eje $y$. Los símbolos abiertos representan zonas de estricción localizada y/o fractura, determinando la posición de la CLC (línea continua) sobre el diagrama. Los diferentes ensayos permitieron obtener el DLC en su totalidad. En el caso del acero DP-780 el mínimo valor de conformabilidad se encuentra bajo condiciones de deformación plana $\left(\rho=\varepsilon_{2} / \varepsilon_{1}=0\right.$ ) con $\varepsilon_{1 \mathrm{~min}} \approx 0,15$; mientras que los valores máximos fueron medidos para tracción uniaxial con $\varepsilon_{1 \max } \approx 0,29$. La chapa de zinc Zn20 muestra un comportamiento inusual sobre el sector derecho del DLC. Las deformaciones límite entre trayectorias de deformación $\rho=0,2$ y $\rho=0,7$ son elevadas, alcanzando su má- 
ximo para $\rho=0,7$ con $\varepsilon_{1 \max } \approx 0,55$. A partir de este punto la formabilidad del material disminuye hasta encontrar su mínimo bajo tracción equibiaxial, $\varepsilon_{1 \text { min }} \approx 0,27$. Este perfil inusual en el rango biaxial es el resultado de las influencias combinadas de la fuerte anisotropía de la aleación de zinc y de la direccionalidad introducida por la fase intermetálica $\mathrm{TiZn}_{16}$ la cual muestra un fibrado en la dirección de laminado. Resultados similares fueron encontrados por MILESI et al. [13] para una chapa de una aleación semejante con un espesor de $1 \mathrm{~mm}$.

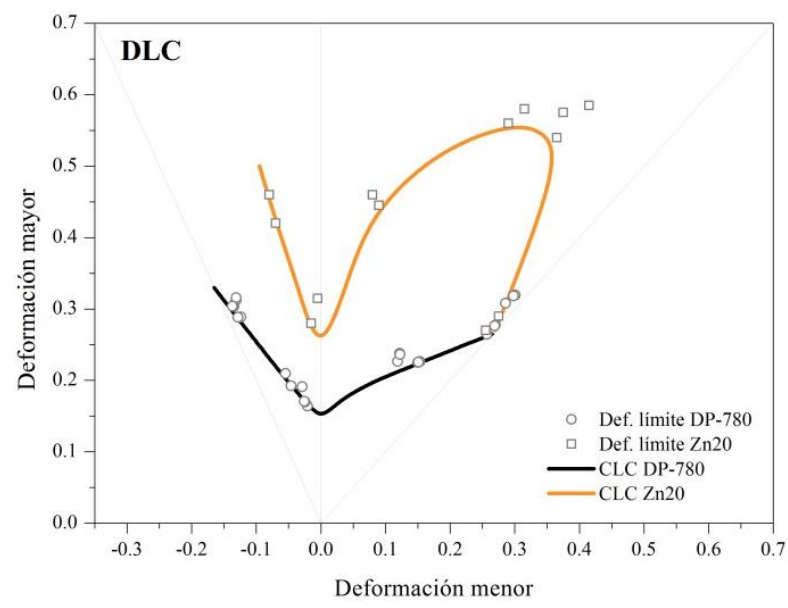

Figura 5: DLC de las chapas de acero DP-780 y zinc Zn20, obtenidos bajo trayectorias lineales de deformación.

La Figura 6 muestra los valores de deformación límite del acero DP-780 obtenidos a partir de muestras con diferentes niveles de pre-deformación equibiaxial $\left(\varepsilon_{1} \approx 0,09 ; 0,13\right.$ y 0,17$)$, subsecuentemente deformadas mediante tracción uniaxial. Los caminos de deformación son también ilustrados para cada una de las muestras ensayadas. Cuando se comparan estos resultados con la CLC obtenida mediante trayectorias lineales se observa un aumento de las deformaciones límite del acero DP-780, evidenciando una no despreciable dependencia de la formabilidad del material con la historia de carga a la que fue sometido. Por otro lado, las muestras pre-deformadas de zinc $\operatorname{Zn} 20\left(\varepsilon_{1} \approx 0,05 ; 0,15\right.$ y 0,32$)$ presentaron un incremento de formabilidad significativo (Figura 7), incluso en muestras cortadas a partir de una probeta Marciniak ya fracturada, tal como se observa en la Figura 8. En este caso particular, el punto de la CLC lineal para solicitación equibiaxial es inferior al de la mayor pre-deformación (Figura 7), lo cual resultaría ilógico. Sin embargo, esto radica en el hecho de que el material es solicitado bajo dos ensayos diferentes, Marciniak y acopado hidráulico. A diferencia de este último, el ensayo Marciniak procede en el plano, es decir, a medida que la probeta es deformada mantiene su superficie plana, evitando los efectos de la curvatura. Esto puede influir sobre la formabilidad del material, induciendo dispersión en los resultados.

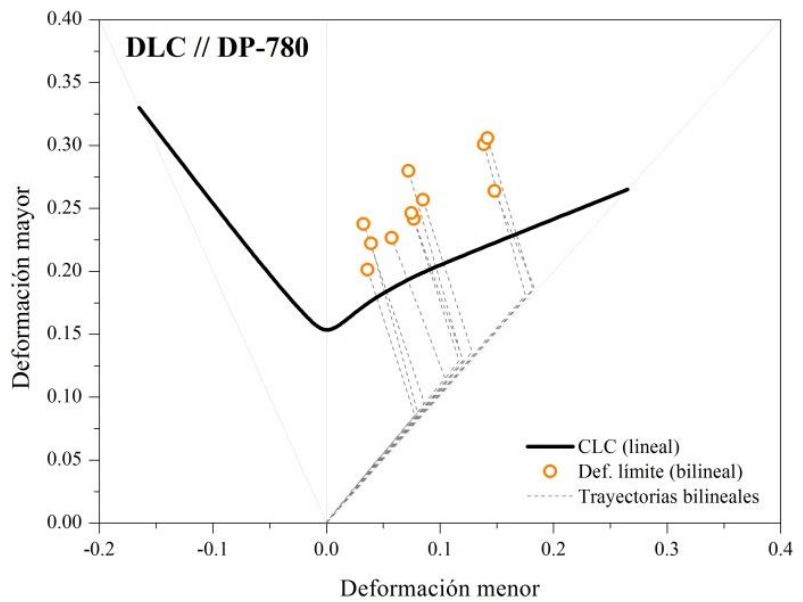

Figura 6: Influencia de trayectorias bilineales de deformación sobre el DLC del acero DP-780. 


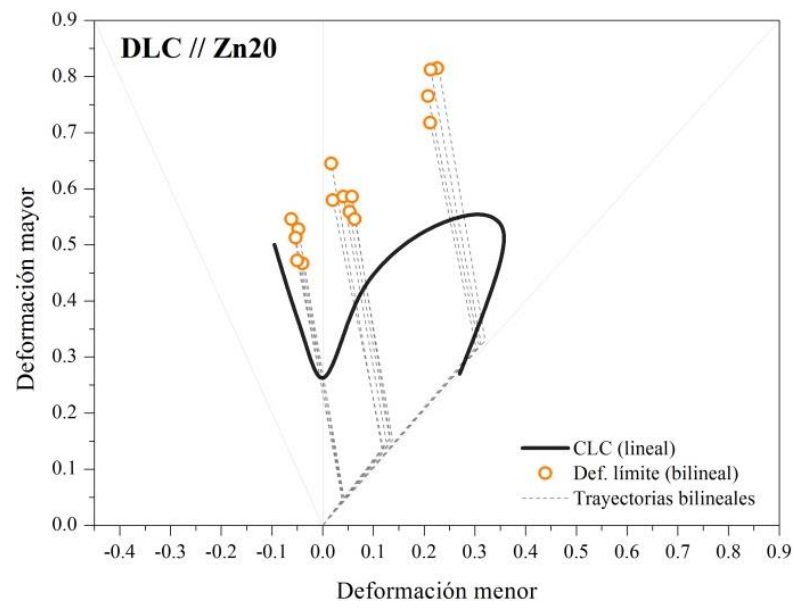

Figura 7: Influencia de trayectorias bilineales de deformación sobre el DLC del zinc Zn20.

Estos resultados se contraponen con estudios reportados en la bibliografía que demuestran que a partir de un determinado nivel de pre-deformación equibiaxial, usualmente pequeño, materiales como aluminio [14-16] y aceros de baja resistencia [15-19] agotan casi inmediatamente su ductilidad durante el subsecuente estirado uniaxial, disminuyendo la CLC sobre el sector derecho del DLC. Mediante el esquema MK en conjunto con un modelo constitutivo anisotrópico de base física, HIWATASHI et al. [18] sugirieron que este efecto es causado por el endurecimiento transitorio que ocurre durante el cambio de trayectoria de deformación, causado por la parte latente de las estructuras de dislocaciones desarrolladas. Este transitorio no fue observado en ninguno de los ensayos realizados, lo cual podría explicar en parte el porqué de los comportamientos observados para los materiales en estudio. Sin embargo, hay que tener en cuenta que en las simulaciones el cambio de trayectoria se da de forma abrupta, sin la descarga del material.

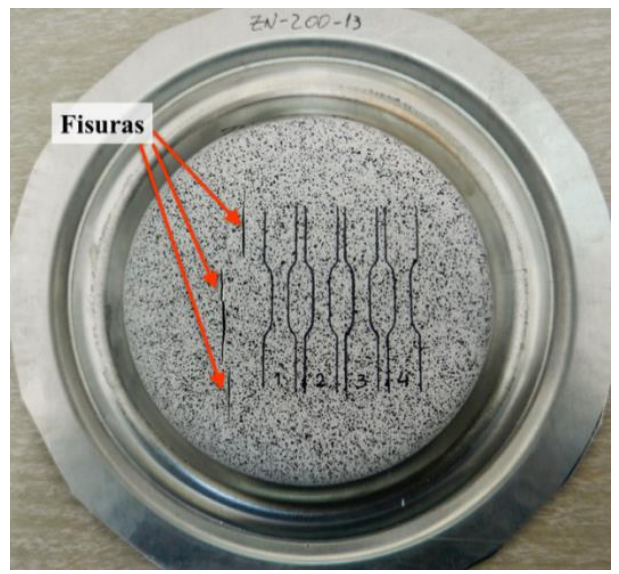

Figura 8: Probetas de tracción uniaxial obtenidas a partir de una muestra Marciniak fracturada de zinc Zn20.

La Figura 9 muestra los valores de deformación límite mayor medidos, en ambos materiales, sobre las probetas de tracción uniaxial y para los diferentes niveles de pre-deformación biaxial. Por un lado, el acero DP-780 muestra una esperada reducción de ductilidad a medida que aumenta el valor de pre-deformación, aunque no tan severa como para disminuir el nivel de la CLC en el sector derecho del DLC. Por otro lado, resulta llamativo que la chapa de zinc muestra un nivel de formabilidad prácticamente constante con el incremento de pre-deformación. Un aspecto importante a considerar para explicar este comportamiento es la influencia de la recristalización dinámica continua (CDRX) experimentada por el zinc, incluso a temperatura ambiente [20]. El zinc, como la mayoría de los materiales hexagonales, retiene una figura de polos basal similar bajo recristalización, es decir, los polos basales muestran estadísticamente la misma orientación, con variaciones menores en intensidad y reorientación de polos prismáticos. Esto fue confirmado por SCHLOSSER et al. [21] para la misma aleación bajo diferentes modos de deformación. Asimismo, la saturación observada en la curva de carga de esta aleación (Figura 2) a partir de los primeros estadios de deformación plástica es compatible con un equilibrio entre el endurecimiento por deformación y la CDRX sufrida por el mate- 
rial, lo cual produce una débil evolución de la microestructura debido al refinamiento de los granos. Esto es objeto actual de estudio, en orden a confirmar su influencia y relación con la deformación límite observada.

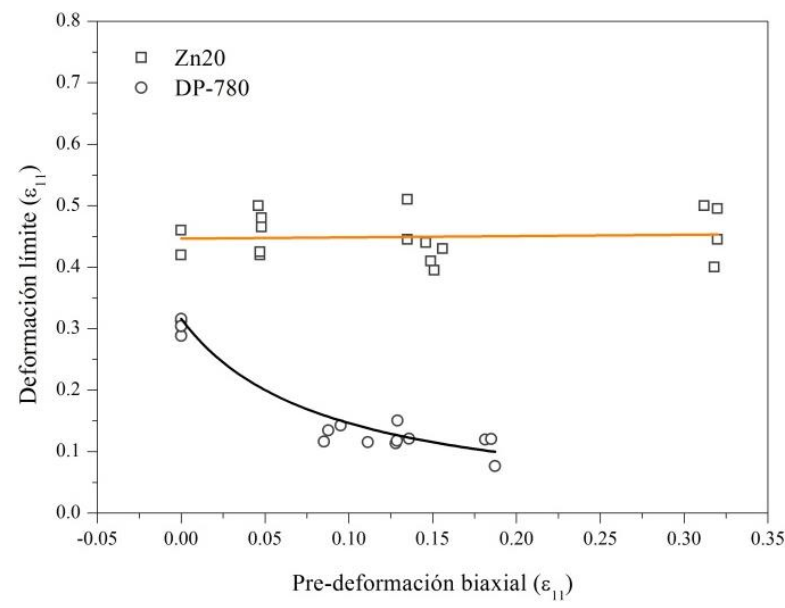

Figura 9: Valores de deformación límite mayor medidos sobre las muestras de tracción uniaxial para los diferentes niveles de pre-deformación biaxial.

\section{CONCLUSIONES}

Se estudió la influencia de los cambios en las trayectorias de deformación sobre la formabilidad de chapas de acero DP-780 y zinc Zn20 mediante ensayos bilineales. En este sentido, los materiales fueron predeformados mediante ensayos Marciniak con el subsecuente corte de muestras de tracción uniaxial de tamaño reducido. A partir de la comparación de estos resultados con las CLCs obtenidas mediante trayectorias de carga bilineales se evidenció una influencia positiva de las pre-deformaciones biaxiales sobre la formabilidad de los materiales ensayados. El incremento en las deformaciones límite fue mayor en las muestras de zinc, lo cual podría deberse a la recristalización dinámica continua (CDRX) que experimenta este material a temperatura ambiente.

\section{AGRADECIMIENTOS}

Este trabajo de investigación fue financiado por la Agencia Nacional de Promoción Científica y Tecnológica, proyecto PICT 2013-0558.

\section{BIBLIOGRAFÍA}

[1] BARATA DA ROCHA, A., BARLAT, F., JALINIER, J.M., "Prediction of the forming limit diagrams of anisotropic sheets in linear and non-linear loading”, Materials Science and Engineering, v. 68, pp. 151-164, 1984.

[2] VOLK, W., HOFFMANN, H., SUH, J., KIM, J., "Failure prediction for nonlinear strain paths in sheet metal forming”, CIRP Annals - Manufacturing Technology, v. 61, n. 1, pp. 259-262, 2012.

[3] SUTTNER, S., MERKLEIN, M., "Characterization of the shear stress state under non-proportional strain paths realized by biaxial stretching in the Marciniak Test", Materials Today: Proceedings $2 S$, v. 2, n. 1, pp. S98 - S106, 2015.

[4] LEOTOING, L., GUINES, D., "Investigations of the effect of strain path changes on forming limit curves using an in-plane biaxial tensile test”, International Journal of Mechanical Sciences, v. 99, pp. 21-28, Ago. 2015.

[5] GRAF, A., HOSFORD, W., "Effect of changing stain path on forming limit diagram of Al 2008-T4", Metallurgical Transactions B, v. 24, pp. 65-75, 1993.

[6] GRAF, A., HOSFORD, W., "The influence of strain path changes on forming limit diagram of A1 6111 T4”, International Journal of Mechanical Sciences, v. 36, pp. 897-910, 1994.

[7] CAO, J., YAO, H., KARAFILLIS, A., BOYCE, M.C., "Prediction of localized thinning in sheet metal using a general anisotropic yield criterion”, International Journal of Plasticity, v. 16, pp. 1105-1129, 2000.

[8] YAO, H., CAO, J., "Prediction of forming limit curves using an anisotropic yield function with prestrain 
induced backstress", International Journal of Plasticity, v. 18, pp. 1013-1038, 2002.

[9] SCHWINDT, C., SCHLOSSER, F., BERTINETTI, M.A., STOUT, M., SIGNORELLI, J.W., "Experimental and Visco-Plastic Self-Consistent evaluation of forming limit diagrams for anisotropic sheet metals: An efficient and robust implementation of the M-K model", International Journal of Plasticity, v. 73, pp. 62 99, 2015.

[10] SUTTON, M.A., ORTEU, J.-J., SCHREIER, H.W., Image Correlation for Shape, Motion and Deformation Measurements, Springer Publishing Company, Incorporated, 2009.

[11] BRAGARD, A., BARET, J.C., BONNARENS, H., "A simplified technique to determine the FLD at onset of necking", Centre Res Metallurgy, v. 33, pp. 53-63, 1972.

[12] SERENELLI, M.J., Textura, anisotropía y formabilidad límite. Una extensión del modelo de MarciniakKuczynski basada en plasticidad cristalina, Tesis Doctoral, Universidad Nacional de Rosario, Rosario, Argentina, 2013.

[13] MILESI, M., LOGÉ, R.E., JANSEN, Y., “Anisotropic mechanical behavior and formability criterion for zinc sheets", Journal of Materials Processing Technology, v. 214, pp. 2869-2876, 2014.

[14] BUTUC, M., BARLAT, F., GRACIO, J., VINCZE, G., "A theoretical study of the effect of the double strain path change on the forming limits of metal sheet", Key Engineering Materials, v. 554-557, pp. 127138, 2013.

[15] LAUKONIS, J.V., GHOSH, A.K., "Effects of strain path changes on the formability of sheet metals", Metallurgical Transactions A, v. 9, n. 12, pp. 1849-1856, Dic. 1978.

[16] MANOPUlO, N., HORA, P., PETERS, P., GORJI, M., BARLAT, F., “An extended Modified Maximum Force Criterion for the prediction of localized necking under non-proportional loading", International Journal of Plasticity, v. 75, pp. 189-203, Dic. 2015.

[17] GOTOH, M., "A class of plastic constitutive equations with vertex effect-IV. Applications to prediction of forming limit strains of metal sheets under nonproportional loadings", International Journal of Solids and Structures, v. 21, n. 12, pp. 1149-1163, 1985.

[18] HIWATASHI, S., VAN BAEL, A., VAN HOUTTE, P., TEODOSIU, C., "Prediction of forming limit strains under strain-path changes: Application of an anisotropic model based on texture and dislocation structure", International Journal of Plasticity, v. 14, n. 7, pp. 647-669, 1998.

[19] KURODA, M., TVERGAARD, V., "Effect of strain path change on limits to ductility of anisotropic metal sheets", International Journal of Mechanical Sciences, v. 42, n. 5, pp. 867-887, 2000.

[20] PHILIPPE, M. J., “Texture formation in hexagonal materials", Materials Science Forum, v. 157-162, pp. 1337-1350, 1994.

[21] SCHLOSSER, F., SCHWINDT, C., FUSTER, V., et al., "Estudio de la evolución de la textura cristalográfica de una chapa de zinc sometida a distintos tipos de deformación". En: $15^{\circ}$ Congreso Internacional de Metalurgia y Materiales SAM-CONAMET, ID147-T4, Concepción, Chile, 17-20 de Noviembre, 2015. 